\title{
Converter induced resonances in microgrids due to high harmonic distortion
}

\author{
N. Etherden ${ }^{1}$, Martin Lundmark ${ }^{2}$, Josep Maria Fernández ${ }^{3}$ and M. H. J. Bollen ${ }^{1}$ \\ ${ }^{1}$ STRI AB \\ Regnbågsgatan $8 \mathrm{~b}$ \\ Gothenburg, 41755 (Sweden) \\ nicholas.etherden@stri.se,math.bollen@stri.se \\ ${ }^{2}$ Luleå University of Technology \\ Department of Electric Power Engineering \\ 93187 Skellefteå, Sweden \\ ${ }^{3}$ CINERGIA \\ Pere IV 29-35, \\ 08018 Barcelona, Spain
}

\begin{abstract}
This paper describes the resonance introduced by harmonics amplification and adverse interaction of electronic converters. An observation of undamped oscillation leading to instability in a microgrid is described. The term "converter induced resonances" is proposed to describe this phenomenon.

The amount of distributed generation, active loads, FACTS and battery energy storage systems are expected to increase in future Smart Grids. All these resources will be interfaced with electronic converters. The potential impact of converter induced resonances in such grids is described.
\end{abstract}

A coordinated design of the control systems of all converters is in practice not feasible. Each device will be independently tested to fulfil grid codes and have its own converter control implemented that can include functionality to modify voltage and /or current waveform

\section{Key words}

Microgrids, power electronic converters, power system harmonics, photovoltaics, battery storage

\section{Introduction}

With the presence of multiple power-electronic converters, situations can occur where the harmonics are amplified due to the interaction between converters. Normally, resonances in the power grid are between passive components like inductors and capacitors. Amplification of harmonic distortion due to such resonances has been the subject of many studies, also in the context of large scale integration of distributed generation $[1,2,3,4,5]$. Devices like solar PV inverters include active power electronic converters. Although the aim of each individual controller is to minimize harmonic levels the presence of multiple power electronic converters can lead to situations where the harmonics are amplified due to the interaction between the converters and their controllers [5].

Many Smart Grid visions of the future power systems anticipate large amounts of distributed generation connected at distribution level, active loads and storage units containing active power electronic converters as interfaces with the grid. Flexible Alternating Current Transmission System (FACTS) are expected to increase and become present also on medium voltage levels. Each converter uses it own control system tested to ensure stable operation under its expected operation environment. Such devices are often tested against a passive grid (in circuit theory terms: a voltage source behind impedance). In reality, other control systems are present as well, either in the grid itself or in other equipment connected to the grid. The presence of multiple control system could result in interaction between them, resulting in undamped oscillations. The term "electronic instability" has been used to refer to such a situation[6], [7]. The oscillations will not in all cases result in an unstable situation leading to an interruption of the power supply. Therefore the more general term "converter induced resonances" is here proposed to describe this phenomenon.

One explanation put forward to describe this resonance is a combination of the active Power Factor Correction (PFC) converters nonlinear behaviour and the choices of parameter values in the regulator [8]. For example [9] reports that grid connected microgrid system with three distributed generation units under certain loading can experience large undamped oscillations that will cause instability when no proper control techniques are used. Further examples of high harmonic levels or instable 
operation are shown in $[10,11,12]$. Experience also shows that it is challenging to drive load with a lot of power electronics from a local generator.

In normal operation of the grid, the voltage at the terminals of equipment is mainly determined by the grid and this will limit the impact of adverse interactions between control systems. The main risk of converter induced resonances occurs during island operation of part of the grid with a number of power electronic inverters (also known as "microgrid operation"). This is where converter is expected to occur first. According to [7] "if a large amount of power is fed to the grid through converters and an increasing part of the load uses inverters, then electronic instability of the entire grid cannot be excluded". Such a situation becomes more likely in a weak grid, i.e. a grid with high source impedance. Even if the source impedance is low at power system frequency (50 or $60 \mathrm{~Hz}$ ) it might be high at harmonic frequencies due to resonances between capacitance and inductance.

In principle such adverse interaction can be avoided in the design stage of the control system, e.g. by proper tuning of the various active and passive filters [13]. However, that requires a coordinated design of the control systems of all converters. This is in practice not feasible, as there are many different manufacturers involved and the number of combinations is too large to test them all.

While the risk of converter induced resonance should not be exaggerated, the combination of off-the-shelf inverters in future building automation or Smart Grid systems may see an increased frequency of occurrence of such phenomena. In the case described in this paper, the oscillation could be mitigated by modification of one of the inverter control schemes. When used together in the hundreds or more the same equipment might exert a stabilizing influence [14], yet it is a concern how to verify such multi-inverter interactions without joint testing.

\section{Experimental microgrid}

The Smart Grid Research, Development and Demonstration (RD\&D) is a three converter microgrid possible of operating in both island and grid connected modes, see Figure 1.

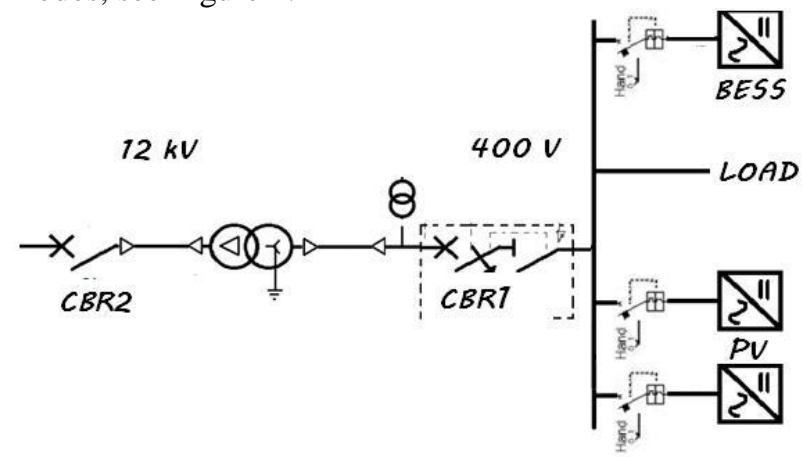

Figure 1 Set-up of $30 \mathrm{kVA}$ rated PV and BESS system. PV inverter works autonomously. For microgrid synchronisation the BESS measures voltage on the low voltage size of the transformer. The BESS is the voltage source of the microgrid and controls the CBR1 breaker, enabling island operation.

\section{PV inverter control}

Solar PV inverters are implemented with active power electronic converters in order to adjust frequency and current wave form to match that of the connected electrical grid. These inverters generally use pulse width modulation (PWM) controllers to generate sinusoidal output currents [5]. They will have multiple control loops to perform a variety of tasks. For a self-commutated voltage source converter the task traditionally include maximum power point tracking (MPPT), reactive power compensation, harmonic cancellation and protection against islanding [5]. Some inverters use the waveform shape of the supply voltage as a reference source. Since the controller wants the converter to behave as a resistance, the controller will force the current to follow the voltage profile. Harmonics in the voltage then provides harmonics in the PV output current.

Only a stable electricity grid will permit large scale expansion of renewable energy. To maintain stability in a grid with large amounts of renewable electricity production also those production units will need to contribute to keeping the grid stable. As the proportion of production from $\mathrm{PV}$ has increased, countries like Germany (through e.g. Art. 6 of the 2009 German Renewable Energies Act, EEG) have required that PV inverters are able to provide also ancillary services. The services implemented in PV systems include:

- autonomous and automatic power reduction based on locally measured increase of frequency

- possibilities for larger plants for remote controlled power reduction from the grid operator in the case of overload.

Static voltage support based on reactive power is also possible to either:

- meet a fixed power factor defined by the grid operator

- meet a power factor transmitted via a communication interface, adapted to the momentary needs of the grid

- control of reactive power based on a predefined characteristic curve.

In these ways the reactive power from PV inverters is used to reduce undesirable voltage increases in low and medium voltage grids. Also Low Voltage Ride Through (LVRT) functionality is being required, implying that the PV units remain connected to the grid during voltage drops and even support the grid by feeding reactive power during the disturbance. As the number and complexity of ancillary services increase also the complexity of the control algorithms of the PV inverters increase.

The two $15 \mathrm{kVA}$ solar PV inverters in the RD\&D are three-phase transformerless grid tie inverters from a major supplier. Typically such a grid tie inverter has an on-board computer which will sense the voltage waveform of the grid and will inject current according to the available active power and the external reactive power commands to meet this waveform. In order to have a feedback of the current injected, the on-board computer 
will measure the current as well. This potentially creates a feed-back loop.

The PV inverter includes grid support and several integrated grid management functions mainly in the form of reactive power provision which is in the RD\&D used to keep the power factor near unity. The reactive power control capabilities of the PV inverters are activated and aimed at keeping the power factor near unity. Further information on the control of the PV inverter is not revealed by the manufacturer.

\section{BESS control}

The Battery Energy Storage System (BESS) Converter is based on two Voltage Source Converters (VSC) of 50 $\mathrm{kVA}$. The converter structure is based on a back-to-back three phase converter. One side of the converter is connected to the $20 \mathrm{kWh}$ of Lithium-ion. The other side is connected to the grid through inductor filter and isolation transformer, see Figure 2. A LCL filter configuration is used as well to interface the grid. The capacitor in the output filter is required in microgrid mode in order to create a sinusoidal voltage output wave form when the microgrid has no load. The LCL configuration has shown to improve dynamic response and filtering action better than single inductor type [13]. The use of LCL filters also removes high order harmonics due to switching of VSC and allows controllers to shape output current and voltage [13]. The value of the capacitors used to filter the AC output depends on the maximum allowable harmonic content on the grid side in connected operation. These capacitors will also decrease the power factor consumed by the transformer without load. However, the use of capacitors will also introduce a resonance frequency in the filter. During the designing stage, this resonance is placed between the maximum controllable frequency and the switching frequency of the converter.

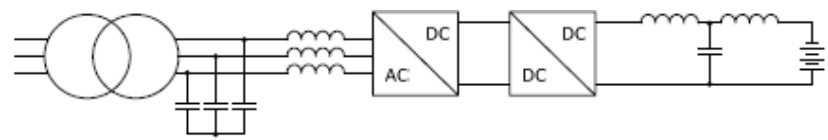

Figure 2 BESS Converter and grid connection diagram.

The DC-to-DC converter is a buck-boost converter that attempts to maintain a constant DC bus voltage on its output while the inverter is drawing a current that is in phase with, and at the same frequency as, the grid voltage. Thus the grid establishes voltage and frequency on the microgrid. In this case, the converter acts like a rotary machine connected to the grid, modifying the prime mover affects to real power and controlling the excitation of the machine is possible to set the reactive power injected.

The converter switching technology is based on Insulatedgate bipolar transistors (IGBTs) ensuring the bidirectional capabilities needed for both active and reactive mode as well as enabling island operation possibilities.

The control strategy chosen when the BESS is connected to the grid mode is a traditional inner current control loop with an external power loop. The direct current component of the inner loop will affect to the real power consumed/injected to the grid and the quadrature component to the reactive power. This control strategy requires knowing the grid phase at each sampling time with precision. For achieving this issue a Phase Locked Loop (PLL) is also implemented, see Figure 2.

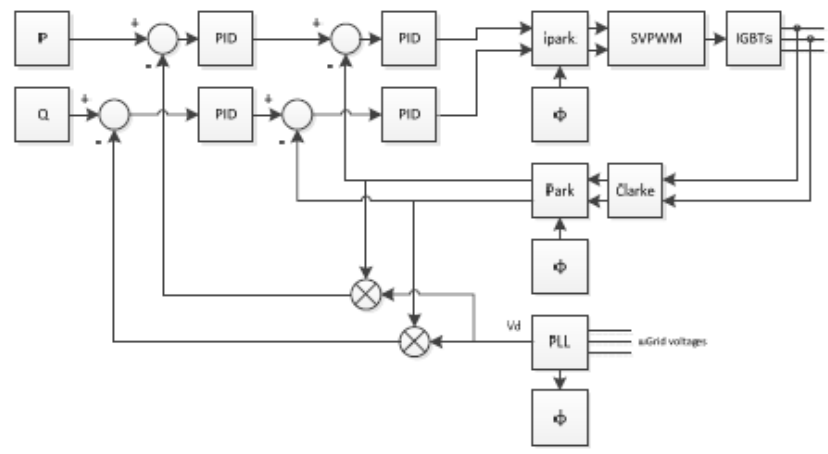

Figure 3 Control diagram for grid connected mode.

In islanded mode, the BESS converter connected to the microgrid will set the voltage and frequency. Under unbalanced and nonlinear loads, the current harmonics can distort the voltage wave at the output of the converter. The control scheme tries to compensate unbalances in the microgrid voltage when it is in this mode and up to 13th harmonic. This control strategy is based on Clarke and Park transformations, PID (Proportional Integral Derivative) controllers, low pass and finally a notch filters to extract the voltage harmonics. The control scheme in island mode is depicted in Figure 4.

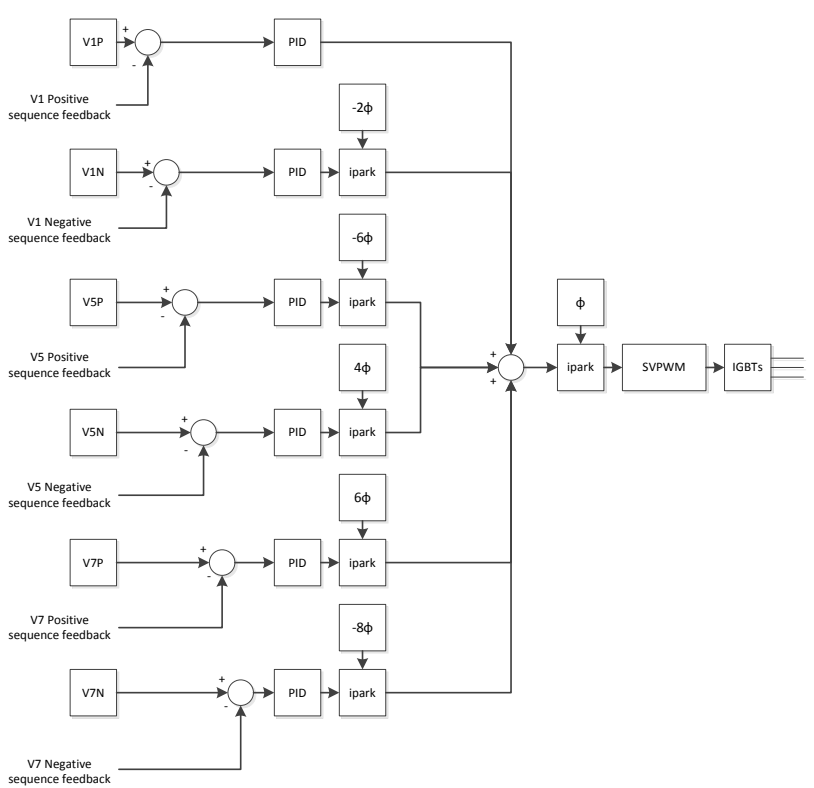

Figure 4 Basic control scheme for islanded operation.

From the voltage measurements and the phase angle generated internally, voltage measurements are transformed in a D; Q coordinates system and then rotated synchronously with the microgrid phase. With these transformations, the voltages $\mathrm{V}_{\mathrm{d}}$ and $\mathrm{V}_{\mathrm{q}}$ appear constant in a rotating coordinates system. The harmonic content of the measured voltages is also rotated but with a different frequency in the transformed coordinate 
system, making it possible to extract the different harmonic content and sequences from the fundamental frequency. The notch filter is needed for doing this separation. Having all the components linearised and represented in $\mathrm{d}$ and $\mathrm{q}$ coordinates system it is possible to apply a traditional PID controller over each component. The output of each PID controller has to be anti-transformed, accumulated with the other PIDs outputs and got into the antitransformation. The output of the current controller is anti-transformed one more time at $50 \mathrm{~Hz}$ before applying a Space Vector Pulse-Width Modulation (SVPWM) algorithm and finally transforming the output into a sinusoidal form.

In order to simplify the scheme, the $\mathrm{d}$ and $\mathrm{q}$ values for each voltage and current have been represented with a single line diagram in Figure 5.

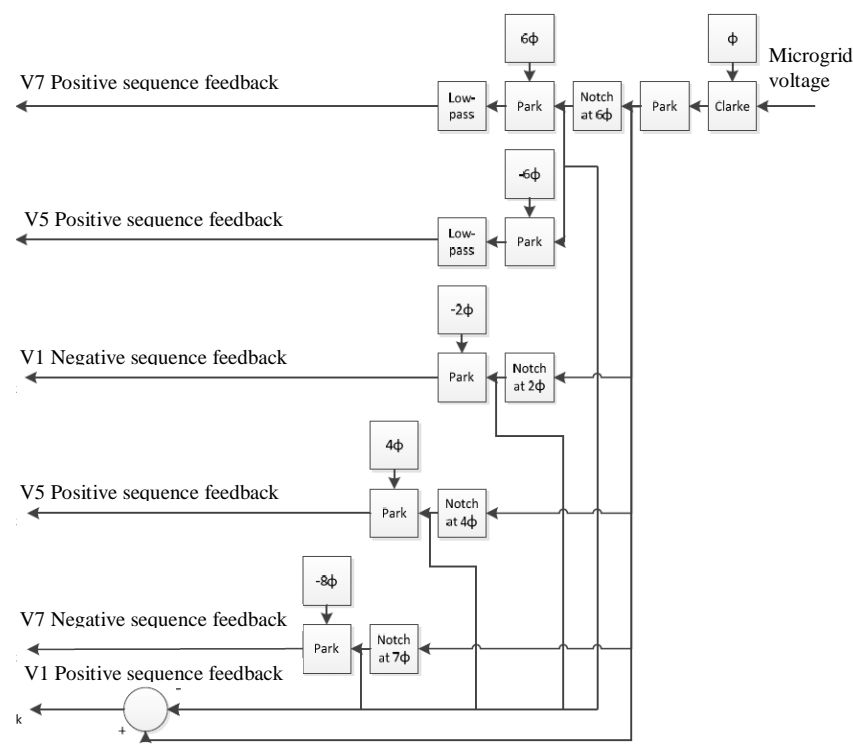

Figure 5 Filters and rotations of harmonic loop control.

An example of the harmonic filtering is given in Figure 6.

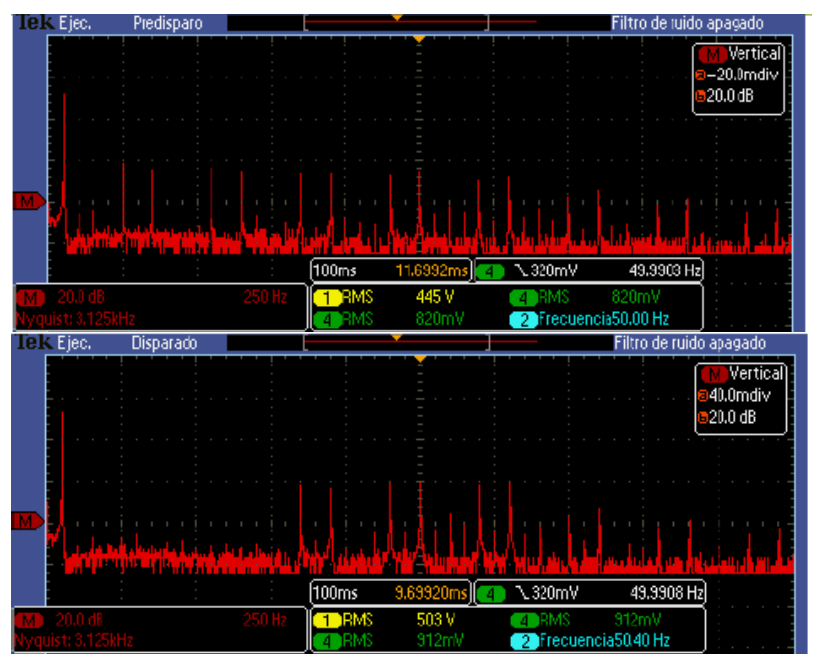

Figure 6 Spectrum of voltage in island operation without (top) and with (bottom) harmonic filtering up to 11th harmonic.

\section{Observations}

The aim of the harmonic filtering algorithm described in the previous section is to keep the voltage in the microgrid sinusoidal during island operation. Still, high levels of harmonic distortion were observed when the BESS and the PV installation where operated together in island mode. The instability was first observed when the PV converters where injecting $10 \%$ of rated power or approximately $3 \mathrm{kVA}$ ( $1.5 \mathrm{kVA}$ each). At such low power output the generated current waveform from the PV is highly distorted according to Figure 7.

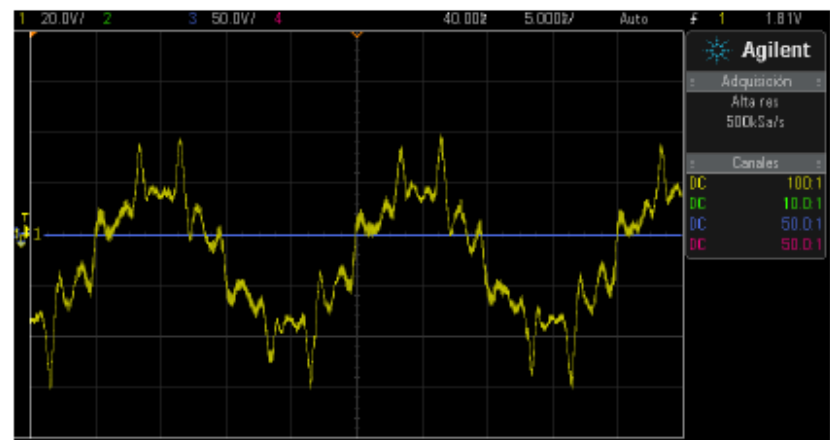

Figure 7 Current injected by PV during grid connected operation and low power production (10\% of rated power). As can be observed, current is highly distorted at such low power production.

The voltage waveform during island operation, when the $2^{\text {nd }}, 4^{\text {th }}$ and $5^{\text {th }}$ harmonic are filtered are given in Figure 8.

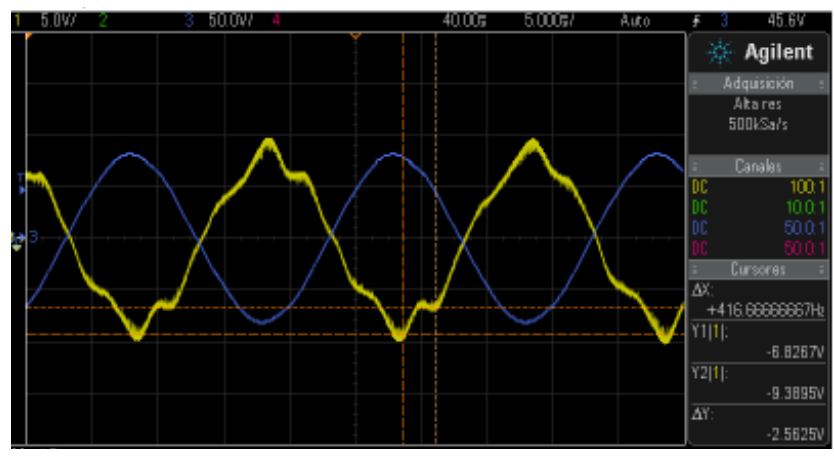

Figure 8 Voltage (blue) and Current (yellow) with PV in island operation. PV is producing at $10 \%$ at rated power and BESS converter is filtering $2^{\text {nd }}, 4^{\text {th }}$ and $5^{\text {th }}$ harmonic. Current and voltage distortion in the microgrid is low compared to Figure 7.

With the harmonic filtering algorithm of the BESS set to filter higher order harmonics ( 7 th to $13^{\text {th }}$ ) the microgrid system becomes instable. The electronic converters interact, effectively seeing each other as disturbance and trying to compensate for each other's contribution. This converter interactions constitutes in practice a "war of the converters" leading to converter induced instability as shown in Figure 9. Without disconnection of the PV converters the oscillation in the 7 th harmonic lead to instability and grid collapse in island operation within a matter of seconds.

In the case of resonances between passive components like inductors and capacitors the addition of a resistor generally helps to add some damping and reduce the distortion. Connection between phases of a $6 \mathrm{~kW}$ resistor 


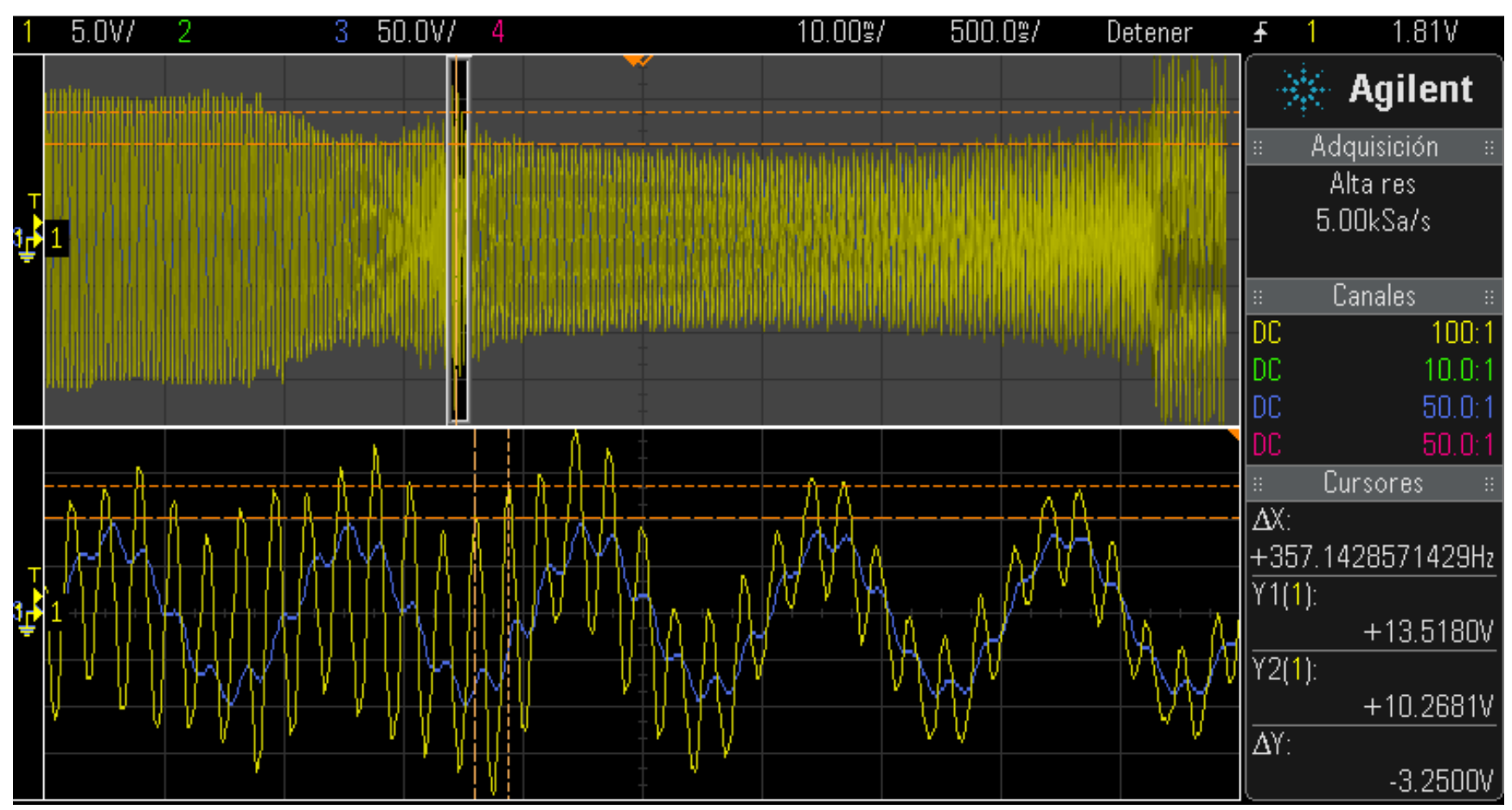

Figure 9 Current with PV in island operation when 2nd to 13th harmonic are filtered by BESS. The interaction now results in high level of 7th harmonic resulting in resonance leading to converter induced resonance and eventual collapse of the microgrid.

(twice the produced power from the PV) did not result in any observable improvement. This indicates that the resonances due to converter interaction behave different than the resonances from passive components.

In effect the two inverters control algorithms see each other as disturbance and try to compensate for each other's voltage and current profile modifications resulting in an oscillation. The oscillation was in this case resolved through modification of the control loop of the BESS, however with commercial converters integrated in future home, building energy automation systems or Smart Grids the converter control algorithms are not likely to be accessible for external partners, making such oscillations difficult to suppress.

\section{Stability}

It is shown that converter induced resonances can occur in a microgrid during island operation. Interaction and resonances in parallel converter systems have been described [15] where line currents can be severely distorted even when the control schemes and filter circuits are properly designed for a single converter system [16]. Such resonances occurring also in future Smart Grids with thousands of off-the shelf power converters cannot, directly, be ruled out.

Resonance between the grid and LCL filter of the converters is a known source of instability [17] and must be handled when determining control strategies. Active damping is a solution [18], sometimes referred to as virtual resistance or virtual impedance [16] [19]. While more challenging to implement than filter based damping it also achieves lower losses [16]. Despite resonance in island operation (grid with near zero fault level) there would be no resonance possible for a grid with perfect voltage source (infinite fault level), as the two controllers cannot influence each other in the latter case. This implies that there must be a threshold fault level below which the resonance occurs. If this stability limit could be quantified in terms of source impedance an approach similar to the hosting capacity method could be applied [20] [21].

\section{Discussion}

The implementation of a stable and well functioning control algorithm is important and the effect of ill conceived control schemes may be revealed unexpectedly. How the best practice of converter control can be transferred to minimum requirements on behaviour of single DER components is a question for further investigation. When there are 1000 different converters in a low-voltage grid), there are 1000*999/2 (about half a million) possible interactions between two converters and $2^{1000}$ (about $10^{300}$ ) possible interactions in total. Testing all possibilities is not practical, yet some degree of verification is required that instability will not occur or is sufficiently unlikely.

In electrical grids with vast amounts of distributed energy resources testing of electronic converter behaviour and stability will be required to ensure interoperability. Requirements and testing should be part of the product standards for DER as well as for normal end-user equipment with active converters. If problems should occur, like equipment malfunctioning, damage, or reduced lifetime, when the number of convert controlled units on a grid increases, some adjustment may be needed as was done in the case described in this paper. 


\section{Conclusion}

Interaction of converters in a $30 \mathrm{kVA}$ rated Smart Grid Research Development and Demonstration (RD\&D) platform built with commercially available solar PV and battery storage converters have been experimentally shown to create oscillations when highly distorted current waveform is injected. The oscillation consisted of extreme high levels of voltage harmonics. The oscillations, as shown in this Figure 9 of this paper, have the potential to cause instability and subsequent collapse of the microgrid. The oscillations could not be damped by the insertion of a resistance, indicating that the resonances due to converter interaction behave different than the resonances from passive components.

Distributed generation units, active loads, SVCs and future battery energy storage systems (BESS) within future Smart Grids are all likely to interface the grid with electronic converters. Each device will be independently tested to fulfil product standards, grid codes and have its own converter control implemented that possibly includes functionality to modify voltage and /or current magnitude and/or waveform. As the number of converters increases, and the complexity of their converter control increases to meet new grid code requirements, the possibilities of adverse interaction between converters increase. The term "converter induced resonances" is proposed for the amplification of harmonic distortion due to such adverse interaction.

The results from trials in our RD\&D confirm that these risks are real. It is unclear to the authors to what extent such oscillations could occur in larger grids. Theoretical studies are required to investigate whether such behaviour could occur also in low or medium voltage grids with very high penetration levels of distributed generators together with other converter control devices. It is further important to study how different parameters impact the risk and severity of the converter induced resonances. The impact of resistive load should be an important part of such studies. The initial results from the experiments show that the impact of resistive load is limited.

\section{Acknowledgement}

This work was undertaken within a joint development project at the HVV (www.highvoltagevalley.se) titled "Smart Grid Energy Storage". Financial support from the Swedish Governmental Agency for Innovation Systems, STRI and STRI AB is gratefully acknowledged.

\section{References}

[1] J. Boemer, M. Gibescu and W. Kling, "Dynamic models for transient stability analysis of transmission and distribution systems with distributed generation: An overview," in IEEE PowerTech, Bucharest, Romania, 2009

[2] W. Wiechowsk and P. Eriksen, "Selected studies on offshore wind farm cable connections - challenges and experience of the Danish TSO," in Power and Energy Society General Meeting Conversion and Delivery of Electrical Energy in the 21st Century, Pittsburgh, USA, 2008.

[3] L. Jun, N. Samaan and S. Williams, "Modeling of large wind farm systems for dynamic and harmonics analysis," in IEEE PES Transmission and Distribution Conference and Exposition, T\&D, Chicago, USA, 2008.
[4] J. Balcells and D. Gonzalez, "Harmonics due to resonance in a wind power plant," in International Conference on Harmonics And Quality of Power, Athens, Greece, 1998.

[5] J. Enslin and P. Heskes, "Harmonic Interaction Between a Large Number of Distributed Power Inverters and the Distribution Network," IEEE Transactions on Power Electronics, vol. 19, no. 6, pp. 1586-1593, 2004

[6] C. Lundmark, A. Larsson and M. Bollen, "Harmonics and highfrequency emission by small end-user equipment," in International Conference on Harmonics and Quality of Power, Cascais, Portugal, 2006.

[7] Å. Larsson, "Power Quality of Wind Turbines," $\mathrm{PhD}$ thesis, Chalmers University of Technology, Gothenburg, Sweden, 2000.

[8] C. K. Tse, O. Dranga and H. H. Iu, "Bifurcation analysis of a power-factor correction boost converter: Uncovering fast-scale instability," in ISCAS '03. Proceedings of the 2003 International Symposium on Circuits and Systems, Bangkok, Thailand, 2003.

[9] W. Al Habri and Y. Magid, "Power system stabilizer for power sharing control of parallel inverters in a grid - Connected microgrid system," in ISGT Middle East, 2011 IEEE PES Conference on Innovative Smart Grid Technologies - Middle East, Jeddah, Saudi Arabia, 2011.

[10] M. Liserre, R. Teodorescu and F. Blaabjerg, "Stability of photovoltaic and wind turbine grid-connected inverters for a large set of grid impedance values," IEEE Transactions on Power Electronics, vol. 21, no. 1, pp. 263-272, 2006.

[11] C. Xin and S. Jian, "Characterization of Inverter-Grid Interactions Using a Hardware-in-the-Loop System Test-Bed," in IEEE 8th International Conference on Power Electronics and ECCE Asia (ICPE \& ECCE), Jeju, Korea, 2011.

[12] M. Cespedes and S. Jian, "Renewable Energy Systems Instability Involving Grid-Parallel Inverters," in Twenty-Fourth Annual IEEE Applied Power Electronics Conference and Exposition, APEC, Washington, USA, 2009.

[13] Y. Mohamed and E. El-Saadany, "Adaptive Decentralized Droop Controller to Preserve Power Sharing Stability of Paralleled Inverters in Distributed Generation Microgrids," IEEE Transaction on Power Electronics, vol. 23, no. 6, pp. 2806-2816, 2008

[14] C. M. Lundmark, "High-Frequency Noise in Power Grids, Neutral and Protective Earth," Licentiate thesis, Luleå University of Technology, Skellefteå, Sweden, 2006.

[15] J. M. Guerrero, J. Matas, C. M. Luis Garcia de Vicuna and J. Miret, "Decentralized Control for Parallel Operation of Distributed Generation Inverters Using Resistive Output Impedance," IEEE Transactions on Industrial Electronics, vol. 54, no. 2, pp. 9941004, 2007.

[16] J. He, Y. Li, D. Bosnjak and B. Har, "Investigation and active damping of multiple resonances in a parallel-inverter based microgrid.," IEEE Transactions on Power Electronics, vol. 28, no. 1, pp. 234-246, 2013.

[17] E. Twining and D. G. Holmes, "Grid current regulation of a threephase voltage source inverter with an LCL input filter," IEEE Transactions on Power Electronics, vol. 18, no. 3, pp. 888-895, 2003.

[18] R. Teodorescu, M. Liserre and P. Rodriguez, Grid Converters for Photovoltaic and Wind Power Systems, Wiley, ISBN: 978-0-47005751-3, 2011.

[19] Y. W. Li, "Control and Resonance Damping of Voltage-Source and Current-Source Converters With LC Filters," IEEE Transactions on Industrial Electronics, vol. 56, no. 5, pp. 1511$1521,2009$.

[20] M. H. Bollen and F. Hassan, Integration of Distributed Generation in the Power System, Hoboken: John Wiley \& Sons, Inc., 2011.

[21] N. Etherden, Increasing the Hosting Capacity of Distributed Energy Resources Using Storage and Communication, Luleå Sweden: Licentiate thesis, Luleå University of Technology, 2012.

[22] M. Bollen, F. Sollerkvist, E. Larsson and C. Lundmark, "Limits to the hosting capacity of the grid for equipment emitting highfrequency distortion," in International Conference on Harmonics and Quality of Power, Cascais, Portugal, 2006. 